\title{
Sistema web de bajo costo para monitorear y controlar un invernadero agrícola
}

\author{
Low cost web system to monitoring and control an agricultural greenhouse \\ Marylin Mamani $^{1} \quad$ Marco Villalobos $^{1 *} \quad$ Raúl Herrera $^{1}$ \\ Recibido 2 de septiembre de 2016, aceptado 31 de mayo de 2017 \\ Received: September 2, $2016 \quad$ Accepted: May 31, 2017
}

\begin{abstract}
RESUMEN
Se ha desarrollado un sistema web de bajo costo que permite monitorear y controlar un invernadero. El monitoreo se realiza mediante la captura de variables climatológicas, dentro del invernadero, con sensores y microcontroladores instalados en su interior, cuyos datos son observados por medio de una aplicación web desde internet. El control se realiza mediante la activación de dispositivos adecuados, de alto voltaje, desde el sistema web. Todo esto es posible gracias a la integración de un protocolo que permite realizar la comunicación entre el usuario y los dispositivos electrónicos implantados dentro del medio ambiente que se monitorea desde internet en tiempo real. El sistema fue sometido a las pruebas de acuerdo al estándar IEEE 1012 y en un laboratorio de cultivo de tejidos vegetales. Los resultados fueron favorables y el sistema está completamente operativo. Las pruebas fueron observadas por un ingeniero agrícola. Así mismo, operó las interfaces que ofrece el sistema, quedando satisfecho por las prestaciones y capacidades. El sistema web de bajo costo integra tecnologías emergentes poco explotadas, con las que se pueden construir una variedad de sistemas, debido a las características que estas poseen. Este es el caso de los microcontroladores seleccionados, los que en comparación a otros no requieren módulos extras, por ejemplo, para conectarse a internet ya que integran todo en una sola placa y cada vez son más pequeños, adaptables para desarrollar cualquier proyecto.
\end{abstract}

Palabras clave: Sistema web, monitoreo-control, invernadero-agrícola, bajo-costo.

\begin{abstract}
A low cost web system has been developed to monitoring and control a greenhouse. The monitoring is performed by capturing climatologic variables inside the greenhouse, using sensors and microcontrollers installed inside it, which data is observed through the internet with a web application. The control is achieved by activating the right device, of high voltage, using the web application. This control is possible thanks to the integration of a protocol that allows communication between the user and the electronic devices implanted inside the environment to be monitored from the internet in real time. The system was tested according to the standard IEEE 1012 and inside a laboratory for vegetable's tissue growing. The results were favorable, and the system is actually fully operational. An agricultural engineer observed the tests. In the same way, he used the interfaces offered by the system, where its performance and capabilities were considered satisfactory. This low cost web system integrates emergent technologies underexploited which can be used to build a wide range of systems, because of the characteristics they possess. This is the case of the selected microcontrollers, which compared to other devices of the same kind, do not require of extra modules, such as network connection module, and because it integrates all in one board and they become smaller along the years, making them adaptable to develop any kind of project.
\end{abstract}

Keywords: Web system, monitoring-control, agricultural-greenhouse, low-cost.

1 Área de Ingeniería en Computación e Informática. Escuela Universitaria de Ingeniería Industrial, Informática y de Sistemas. Universidad de Tarapacá. Arica, Chile.E-mail: mary.magda00@gmail.com; rherrera@uta.cl

* Autor de correspondencia: mvillalo@uta.cl 


\section{INTRODUCCIÓN}

Este trabajo surge a raíz de la necesidad generada en el rubro de la agricultura en una zona desértica como lo es la Región de Arica y Parinacota de Chile, donde las áreas de cultivo mediante invernaderos o mallas antivirus no son despreciables, adaptándose nuevos terrenos ubicados en zonas alejadas, a veces de difícil acceso. Como muestra la Tabla 1, desde el 2013 la superficie cultivada en hectáreas, se mantiene por sobre las 2.400 , lo que es significativo considerando que es una zona desértica.

Tabla 1. Superficie cultivada en la región durante el 2007 al 2014 [2].

\begin{tabular}{|c|c|c|c|c|c|c|}
\hline Año & $\mathbf{2 0 0 7}$ & $\mathbf{2 0 1 0}$ & $\mathbf{2 0 1 1}$ & $\mathbf{2 0 1 2}$ & $\mathbf{2 0 1 3}$ & $\mathbf{2 0 1 4}$ \\
\hline Hectáreas & 3.075 & 2.771 & 2.473 & 2.753 & 2.470 & 2.646 \\
\hline
\end{tabular}

Fuente: Extraída de [2].

Según Pier Lombardi: Hace 6 o 7 años había 20 a 30 hectáreas bajo mallas antivirus, ahora se habla de 500 a 600 hectáreas. Ha sido un cambio importante en el pensamiento, en cómo manejar las plantas, en cómo regarlas, fumigarlas y fertilizarlas [1].

Ante esta situación los agricultores muchas veces tienen que contar con personal encargado y entendido en el tema de cultivo, para analizar y controlar el estado de los niveles de agua, temperatura, luz, etc., de los cultivos, y tener que presentarse físicamente en la zona donde se ubica el invernadero para realizar la revisión, generando gastos en movilización y tiempo. Muchas veces el sistema utilizado no permite detectar a tiempo riesgos en los cultivos que más tarde afectan en la cantidad de productos durante la cosecha.

Hoy en día, existen algunas soluciones principalmente extranjeras que resuelven algunos de los problemas mencionados anteriormente mediante el uso de una infraestructura tecnológica, permitiendo un monitoreo ambiental automatizado de los invernaderos pero, debido a su alto costo de inversión para su adopción y difícil adquisición, muchos productores optan por desechar esta opción [3].

En este trabajo se presenta el desarrollo de una solución mediante el uso de tecnologías de bajo costo que permite realizar un monitoreo en forma constante vía web, del estado de riego y las condiciones climáticas en el invernadero, mediante el uso de computadoras y componentes electrónicos (microcontrolador, sensores, etc.), con los que, el agricultor podrá detectar en forma más rápida posibles peligros que puedan afectar su producción y así determinar una decisión, desde cualquier lugar donde se encuentre, ahorrando gastos en movilización y tiempo. Podrá llevar un mejor control de las condiciones climatológicas y riego, aptas para cada tipo de plantas dentro de los invernaderos, utilizando en el caso del agua, la cantidad justa y precisa requerida por el tipo de plantas, para así economizar al máximo este recurso, sobre todo en zonas donde es escaso. Esto permitirá un buen desarrollo de las plantas y con ello una mejor producción, generando mayores ganancias para el agricultor y más disponibilidad de bienes alimenticios para la población, en especial para aquellas que cuentan con un gran número de personas ubicadas en zonas urbanas, que van expandiéndose continuamente. La solución que se propuso es la utilización de sensores de temperatura y humedad (para suelo y aire), instalados dentro del invernadero que conectados a un microcontrolador, permitirá observar los datos capturados en todo momento vía web mediante la aplicación que a su vez mostrará al usuario las alertas que se generen en forma automática.

Por otra parte, este sistema permitirá a los actores integrar el uso de la tecnología en la agricultura, aprovechando los beneficios que esta les provee, como la reducción de los riesgos de pérdida de capital por falta de un minucioso monitoreo y control de las condiciones climatológicas en las plantas, una mejor producción. Esto es posible, sacando provecho a la disponibilidad de conexión a internet que hoy está llegando a casi todos los rincones del país mediante la telefonía móvil.

Para este sistema se seleccionaron dispositivos electrónicos que hoy son emergentes, muy poco explotados y con los que se pueden desarrollar una variedad de sistemas, debido a las características que estos poseen, como es el caso de los microcontroladores seleccionados, quienes en comparación a otros no requieren módulos extras, por ejemplo, para conectarse a internet ya que integran todo en una sola placa y cada vez son más pequeños, 
adaptables para desarrollar cualquier proyecto. También, se seleccionaron tecnologías del área de la informática que están surgiendo de la mano de las tecnologías electrónicas, que buscan llevar a efecto, junto a estas últimas tareas como la automatización, es el caso del protocolo Mqtt. Al integrar dichas tecnologías, se generó un sistema que demostró ser eficaz, de bajo costo y aplicable en lugares donde se requiere llevar un control preciso de condiciones ambientales, como un invernadero agrícola.

En las siguientes secciones se describe el marco teórico asociado con el desarrollo del sistema (invernadero y crecimiento de plantas, monitoreo y sensores) y el trabajo relacionado. Posteriormente se describe el diseño, la implementación, las vistas y pruebas del sistema, los resultados y, finalmente, las conclusiones.

\section{MARCO TEÓRICO}

\section{Invernaderos y crecimiento de plantas}

Un invernadero es ideal para cultivar ya que al ser un recinto cerrado, se pueden proteger los cultivos de ciertas variaciones climáticas y permite generar un clima óptimo. En la Región de Arica y Parinacota de Chile (zona desértica), los sistemas de cultivos protegidos o bajo invernadero han generado un aumento de la producción de hortalizas de invierno, debido principalmente a las ventajas asociadas con las estructuras de protección que inicialmente cumplían un rol de exclusión, para evitar el ataque de insectos o virus. Actualmente son instaladas con diversos fines y materiales, según el cultivo a proteger, principalmente tomate y pimiento utilizando malla antiáfido (malla microperforada que impide el paso de la mosca de la fruta) y polietileno respetivamente [4]. En la región, muchos agricultores están mejorando los procesos de producción con malla antiinsectos o antivirus y una minoría está trabajando con invernaderos más sofisticados [5]. En el 2007 la región contaba con una superficie total de 2.877 hectáreas cultivadas con hortalizas, desglosadas en 2.854 hectáreas al aire libre y 24 hectáreas bajo invernadero (INE, 2007) [2]. Según Pier Lombardi (2014) este último método ha ido en aumento, ahora se habla de 500 a 600 hectáreas [1].

El desarrollo de los cultivos, en sus diferentes fases de crecimiento, está condicionado por el riego y cuatro factores ambientales o climáticos: temperatura, humedad relativa, iluminación, dióxido de carbono $\left(\mathrm{CO}_{2}\right)[6,7]$. Para que las plantas puedan realizar sus funciones, es necesaria la conjunción de estos factores dentro de unos límites mínimos y máximos, fuera de los que las plantas cesan su metabolismo, pudiendo llegar a la muerte.

\section{Monitoreo}

En el ámbito de la agricultura, según Alvarado [8], cuando se realiza un monitoreo efectivo se anticipa a la aparición de posibles problemas. De esta manera, la toma de decisiones es más económica y efectiva. En ocasiones, los datos obtenidos por medio del monitoreo provoca cambios en las prácticas de manejo. El monitoreo posibilita el control y estudio más a fondo del estado climatológico dentro del invernadero. Las variables generalmente utilizadas son la temperatura, humedad relativa e iluminación. Estas pueden ser captadas mediante sensores especializados y los datos, ser enviados a un microcontrolador el que los prepararía para ser remitidos a un computador.

Los avances tecnológicos en la electrónica han permitido el desarrollo de sensores y microcontroladores que pueden ser utilizados en la agricultura permitiendo el aumento de la producción y reducción de costos.

\section{Sensar y sensores}

Sensar es una técnica utilizada para recopilar información acerca de un objeto físico o proceso, incluyendo la ocurrencia de eventos como un descenso en la temperatura o la presión. Un objeto que realiza tal trabajo de sensar se denomina sensor.

Desde un punto de vista más técnico, un sensor es un dispositivo que traduce los parámetros o eventos en el mundo físico a señales que pueden ser medidas y analizadas. Estos sensores también suelen ser comúnmente llamados transductores, los que a menudo son utilizados para convertir energía de una forma a otra. Un sensor, entonces, es un tipo de transductor que convierte energía del mundo físico a energía eléctrica, que puede ser pasada a un sistema computacional o controlador. Existen redes de sensores inalámbricos que incluyen actuadores que les permiten controlar directamente el mundo físico. Por ejemplo, un actuador puede ser una válvula de control del flujo de agua caliente, un motor que abre o cierra una puerta, o una bomba que controla el nivel de combustible inyectado en un motor. 


\section{Trabajo relacionado}

El sistema propuesto por Jianfa et al. [9], fue diseñado para monitorear y controlar la iluminación, temperatura y humedad de un invernadero de MaxBerry (un tipo de árbol subtropical que produce la llamada fresa china) y así obtener una mejor producción y calidad. En la Figura 1, se muestra la infraestructura general del sistema. Este se compone de cuatro subsistemas que se describen en la Tabla 2.

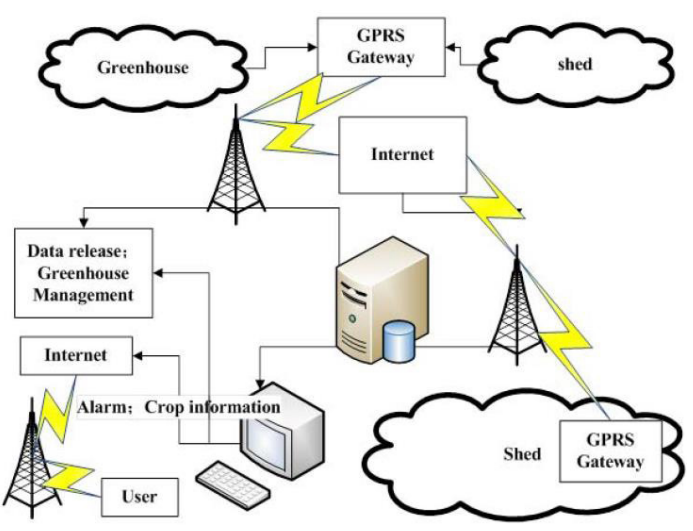

Figura 1. Infraestructura del sistema. Fuente: Extraído de [9].
El proyecto desarrollado por Tongtong et al. [10], consiste en detectar y controlar la temperatura y humedad del medio ambiente de invernaderos para diferentes distancias. En la Figura 2, se muestra la infraestructura general del sistema. Este se compone de un conjunto de elementos que se describen en la Tabla 3.

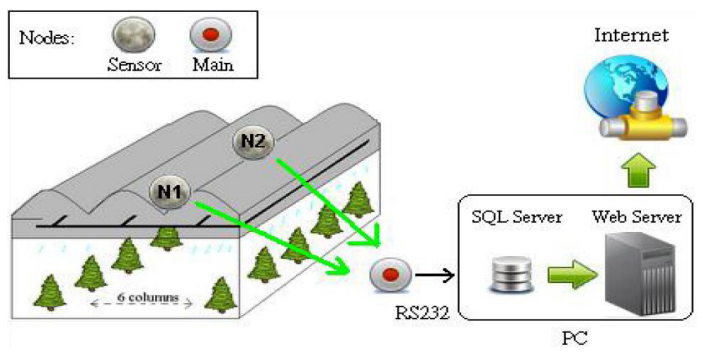

Figura 2. Arquitectura del sistema. Fuente: Extraído de [10].

La propuesta de Balaji et al. [11], consiste en el diseño y desarrollo de un sistema de vigilancia de temperatura, humedad y dióxido de carbono, para aumentar la productividad y la calidad en la agricultura, utilizando una red inalámbrica de

Tabla 2. Subsistemas propuestos en el trabajo de Jianfa et al. [9].

\begin{tabular}{|c|c|}
\hline Subsistemas & Descripción \\
\hline Adquisición de datos & $\begin{array}{l}\text { Basado en redes inalámbricas de sensores (WSN). Se despliega al interior del } \\
\text { invernadero y consiste en un número de nodos sensores inalámbricos que incluyen } \\
\text { sensores de temperatura y humedad. El nodo sensor está basado en el microcontrolador } \\
\text { JN5121 y utiliza los chips MSP 430F1611 y CC2420 para asegurar la versatilidad } \\
\text { y reducir el consumo de energía de los nodos. }\end{array}$ \\
\hline Procesamiento de datos & $\begin{array}{l}\text { Basado en una arquitectura cliente-servidor. Recopila los datos transmitidos por medio } \\
\text { de WSN y los reenvía mediante una interfaz RS232. Luego el módulo GPRS envía } \\
\text { los datos reenviados utilizando la red móvil GPRS a un servidor remoto ubicado a } 5 \\
\text { km de distancia de los invernaderos. En este último, los paquetes de datos recibidos } \\
\text { son divididos para luego almacenar los datos más relevantes en una base de datos. }\end{array}$ \\
\hline Difusión de información & $\begin{array}{l}\text { Basado en la web e integrado con Google Maps. Entregan información sobre el } \\
\text { entorno de producción y los estados, así como la información de usuario, etc. En este } \\
\text { subsistema se integra la técnica de los mapas de Google para entregar reconocimiento } \\
\text { de ubicación. }\end{array}$ \\
\hline Alarma & $\begin{array}{l}\text { Determina varias estrategias de alarma sobre la base de conocimientos y experiencia } \\
\text { relevantes del producto, se establecen umbrales de alarma alternativos para varios } \\
\text { parámetros clave, una vez que los valores de medición de estos parámetros exceden } \\
\text { los umbrales, se envían automáticamente alarmas en formato de voz o SMS por el } \\
\text { sistema a teléfonos móviles designados. }\end{array}$ \\
\hline
\end{tabular}

Fuente: Elaboración con base a [9]. 
Tabla 3. Componentes propuestos en el trabajo de Tongtong et al. [10].

\begin{tabular}{|c|c|}
\hline Componente & Descripción \\
\hline Red de sensores & $\begin{array}{l}\text { Sensores inalámbricos (WSN) con topología en estrella, el que incluye nodos sensores, } \\
\text { algunos nodos de repetición, un nodo principal, un PC terminal, } M y S q l \text {, servicio web y un } \\
\text { sistema de alarma de tiempo real. Los datos de los sensores son transmitidos mediante un } \\
\text { dispositivo móvil y recibidos mediante una señal inalámbrica en el nodo principal. El PC } \\
\text { terminal puede usar el lenguaje Microsoft Visual Basic para programar la lectura de los datos } \\
\text { a través de RS232. Los datos leídos son decodificados y la información útil es almacenada } \\
\text { en la base de datos. El PC terminal utiliza el servidor web para subir los datos a internet. }\end{array}$ \\
\hline Servidor web & $\begin{array}{l}\text { Dibuja una curva para mostrar el cambio de temperatura y humedad en un tiempo limitado. } \\
\text { Cuando la curva cambia de manera anormal, el sistema de alarma informa al trabajador por } \\
\text { vía de correo electrónico, mensaje de texto a su teléfono celular, o puede desencadenar un } \\
\text { sistema de nebulización, abrir respiraderos, encender ventiladores o controlar una amplia } \\
\text { variedad de respuesta del sistema. }\end{array}$ \\
\hline Nodo sensor & $\begin{array}{l}\text { Incluye un módulo sensor HMZ-433A1 para la detección de la humedad, un módulo sensor PT100 } \\
\text { para la detección de la temperatura, el módulo MEGWIN MCU 8051 y un módulo transceptor } \\
\text { inalámbrico NRF2401L. El nodo sensor es controlado por MCU y su principal tarea es recoger } \\
\text { los datos de los sensores analógicos, llevados al módulo ADC de MCU y transformarlos de } \\
\text { datos analógicos a datos digitales. Finalmente, estos últimos datos son cargados y transmitidos } \\
\text { mediante módulo de RF, de 2,4 GHz de NRF2401L hacia el nodo principal. Cuando este recibe } \\
\text { la señal inalámbrica, los datos son transmitidos al PC terminal por conducto del puerto serie, } \\
\text { aquí la información es decodificada y almacenada en la base de datos MySql. }\end{array}$ \\
\hline
\end{tabular}

Fuente: Elaboración con base a [10].

sensores. En la Figura 3, se muestra la infraestructura general del sistema. Este se compone de un conjunto de elementos que se describen en la Tabla 4.

En el trabajo de Guofang et al. [12], se describe un sistema de monitoreo remoto para invernadero basado en la tecnología Zigbee, la herramienta de desarrollo fue LabVIEW 8.6 y Access. En la Figura 4, se muestra la infraestructura general del sistema. Este se compone de un conjunto de elementos que se describen en la Tabla 5.

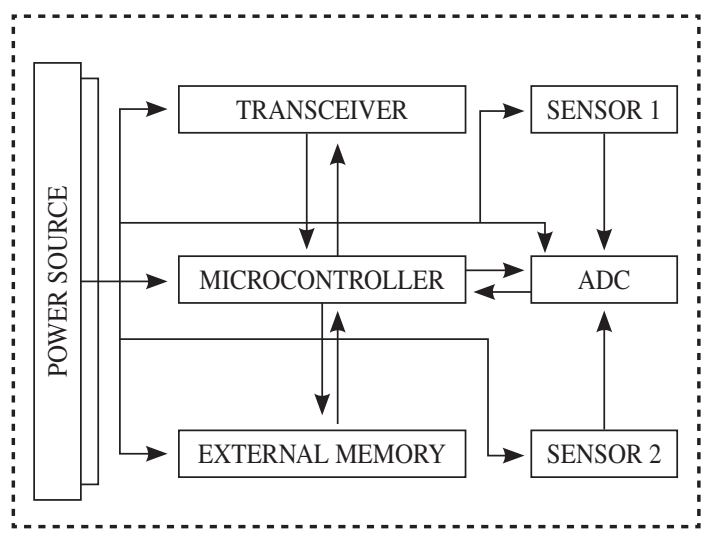

Figura 3: Estructura del nodo hardware.

Fuente: Extraído de [11].
Tabla 4. Componentes propuestos en el trabajo de Balaji et al. [11].

\begin{tabular}{|c|c|}
\hline Componente & Descripción \\
\hline Sensores & $\begin{array}{l}\text { Una topología estrella, donde } \\
\text { cada nodo sensor se comunica } \\
\text { sólo con su puerta de enlace, } \\
\text { enviándole los datos detectados } \\
\text { mediante paquetes de datos, que } \\
\text { luego son almacenados en la } \\
\text { base de datos. }\end{array}$ \\
\hline Aplicación web & $\begin{array}{l}\text { Aplicación web donde los } \\
\text { usuarios se registran y pueden } \\
\text { observar entre otros, las tierras } \\
\text { agrícolas pertenecientes a los } \\
\text { agricultores, el histórico de datos } \\
\text { mediante una vista gráfica y el } \\
\text { estado de los nodos sensores. }\end{array}$ \\
\hline Procesador & $\begin{array}{l}\text { Un procesador ATMEGA8535 y } \\
\text { convertidor analógico a digital } \\
\text { Ic-S8817, como se muestra } \\
\text { en la estructura de hardware } \\
\text { de un nodo en la Figura } 3 . \\
\text { Además WSN utiliza el módulo } \\
\text { transceptor inalámbrico basado } \\
\text { en el protocolo Zigbee. }\end{array}$ \\
\hline
\end{tabular}

Fuente: Elaboración con base a [12]. 


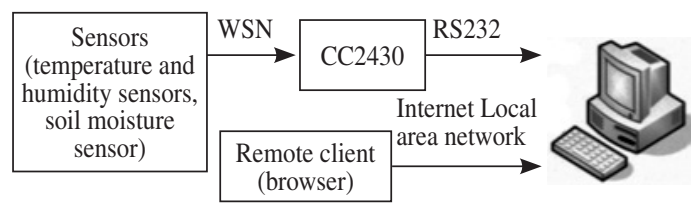

Figura 4 Diagrama esquemático del sistema de monitoreo remoto.

Fuente: Extraído de [12].

Tabla 5. Componentes propuestos en el trabajo de Guofang et al. [12].

\begin{tabular}{|c|c|}
\hline Componente & Descripción \\
\hline $\begin{array}{l}\text { Monitoreo } \\
\text { central }\end{array}$ & $\begin{array}{l}\text { Subsistema de monitoreo del servidor } \\
\text { web que se ejecuta en el invernadero. } \\
\text { El software de monitoreo del servidor } \\
\text { permite obtener información de los } \\
\text { sensores inalámbricos, su función } \\
\text { principal es el ajuste de parámetros, } \\
\text { visualización, consulta, análisis, } \\
\text { reporte de procesamiento y alarma } \\
\text { para los datos en tiempo real, } \\
\text { monitoreo online en tiempo real, } \\
\text { visualización de curva en tiempo real } \\
\text { mediante un gráfico y la comunicación } \\
\text { en tiempo real a distancia. }\end{array}$ \\
\hline $\begin{array}{l}\text { Monitoreo } \\
\text { cliente }\end{array}$ & $\begin{array}{l}\text { Subsistema de monitoreo del cliente } \\
\text { que se ejecuta en la red. Permite } \\
\text { observar la información generada } \\
\text { por el monitoreo central. }\end{array}$ \\
\hline $\begin{array}{l}\text { Adquisición } \\
\text { de datos }\end{array}$ & $\begin{array}{l}\text { Puede adquirir los datos del medio } \\
\text { ambiente en tiempo real y permitir un } \\
\text { monitoreo en forma remota por medio } \\
\text { de internet. La red de adquisición y } \\
\text { transmisión de datos es construida } \\
\text { sobre una red inalámbrica de sensores } \\
\text { y bus RS232. Los nodos sensores } \\
\text { dispuestos en el invernadero son de } \\
\text { temperatura, humedad del aire y de } \\
\text { humedad del suelo. El nodo central } \\
\text { es un chip CC2430, el que recibe } \\
\text { información de cada nodo sensor } \\
\text { en forma inalámbrica, y la carga al } \\
\text { servidor por conducto del Bus RS232 } \\
\text { con el fin de guardar los datos. }\end{array}$ \\
\hline
\end{tabular}

Fuente: Elaboración con base a [12] .

En Collins et al. [13], se describe el desarrollo de un sistema autónomo para monitorear la emisión de gases en un vertedero. Está basado en ciertos componentes alojados dentro de una estructura robusta, como se muestra en la Figura 5. Este

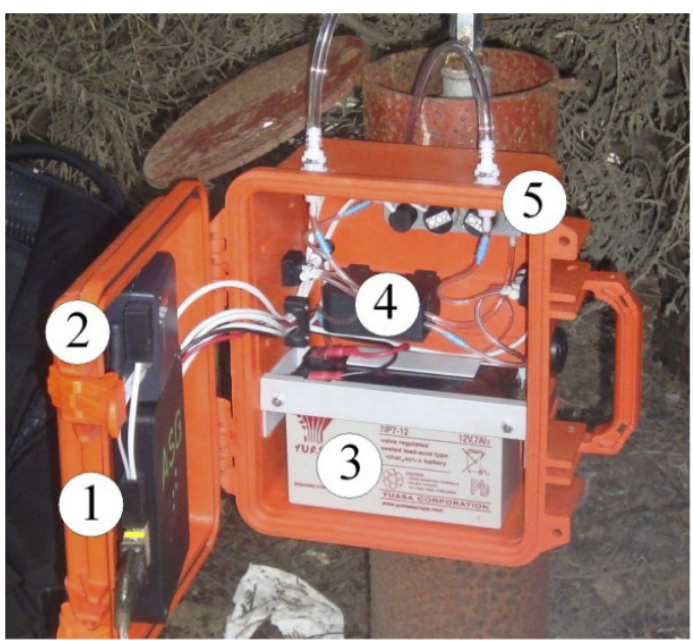

Figura 5. Representación de la plataforma de monitoreo de gas.

Fuente: Extraído de [13].

Tabla 6. Componentes propuestos en el trabajo de Collins et al. [13].

\begin{tabular}{|l|l|}
\hline Componente & \multicolumn{1}{|c|}{ Descripción } \\
\hline Supervisor & $\begin{array}{l}\text { La operación de supervisión se contro- } \\
\text { la mediante un microcontrolador } \\
\text { programado MSP430 (1). }\end{array}$ \\
\hline Comunicador & $\begin{array}{l}\text { Comunicación de datos a través del } \\
\text { módulo GSM (2). }\end{array}$ \\
\hline Energía & $\begin{array}{l}\text { La fuente de alimentación que es } \\
\text { por medio de una batería y permite } \\
\text { un despliegue de 4 semanas a una } \\
\text { frecuencia de muestreo de cuatro } \\
\text { ciclos de muestras por día (3). }\end{array}$ \\
\hline $\begin{array}{l}\text { Actuador } \\
\text { extractivo }\end{array}$ & $\begin{array}{l}\text { La gestión del gas de extracción } \\
\text { por conducto de una bomba de } \\
\text { extracción (4). }\end{array}$ \\
\hline $\begin{array}{l}\text { Muestreador } \\
\text { de gas }\end{array}$ & $\begin{array}{l}\text { El muestreo de gas mediante una cá- } \\
\text { mara de muestra y sensores de } \\
\text { emisiones de gases como dióxido de } \\
\text { carbono }\left(\mathrm{CO}_{2}\right) \text { y metano }\left(\mathrm{CH}_{4}\right)(5) .\end{array}$ \\
\hline
\end{tabular}

Fuente: Elaboración con base a [13].

se compone de un conjunto de elementos que se describen en la Tabla 6.

Mendoza [14], describe un sistema mediante el diseño e implementación de un prototipo que permite controlar y monitorear la temperatura y humedad de un invernadero desde cualquier parte mediante una red LAN. En la Figura 6, se muestra la infraestructura 
general del sistema. Este se compone de un conjunto de elementos que se describen en la Tabla 7.

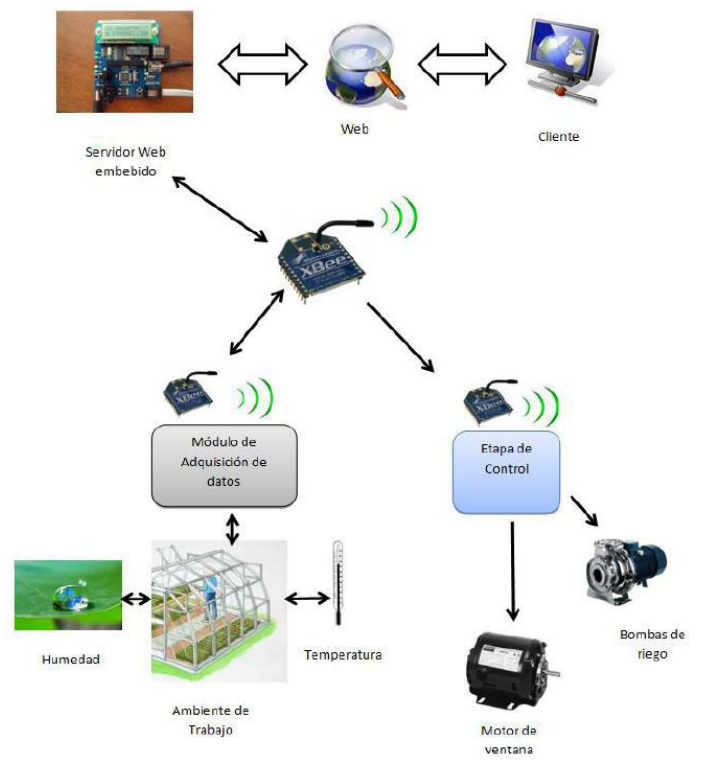

Figura 6. Sistema de control y monitoreo.

Fuente: Extraído de [14].

Tabla 7. Componentes propuestos en el trabajo de Mendoza [14].

\begin{tabular}{|l|l|}
\hline Componente & \multicolumn{1}{|c|}{ Descripción } \\
\hline Micro- & $\begin{array}{l}\text { De alto rendimiento Pic18F4620 que } \\
\text { permite la implementación de un } \\
\text { servidor web embebido o empotrado. } \\
\text { controladores } \\
\text { Permite realizar el monitoreo y } \\
\text { control de las diferentes variables } \\
\text { dentro de un invernadero, mediante } \\
\text { una página web embebida a la que } \\
\text { el cliente puede acceder digitando } \\
\text { en su navegador web la dirección IP } \\
\text { (estática) del sistema, para observar } \\
\text { las mediciones de dichas variables y el } \\
\text { estado de los dispositivos de control. }\end{array}$ \\
\hline Adquisición & $\begin{array}{l}\text { Consta de elementos necesarios } \\
\text { para proporcionar las mediciones } \\
\text { de temperatura y humedad que se } \\
\text { envían al microcontrolador para } \\
\text { ser procesados. Además consta de } \\
\text { un controlador digital de señales }\end{array}$ \\
(DSC), para procesar las señales \\
de los sensores.
\end{tabular}

Fuente: Elaboración con base a [14].

\section{DISEÑO}

\section{Arquitectura del sistema}

La arquitectura define el conjunto de componentes de un sistema, las interfaces de comunicación de los mismos y la forma en que estos componentes se comunican entre ellos, usando dichas interfaces.

El sistema se compone de cinco módulos, que son: módulo de recolección y envío de datos, módulo servidor, módulo control de dispositivos, módulo de monitoreo y control, y el módulo de CloudMQTT.

Los módulos de "recolección y envío de datos" y "control de dispositivos", son circuitos electrónicos basados en microcontroladores, que para el primer caso están conectados a sensores y el segundo, a dispositivos electrónicos de alto voltaje que puede controlar. La interacción entre estos módulos y el resto del sistema se puede observar en la Figura 7.

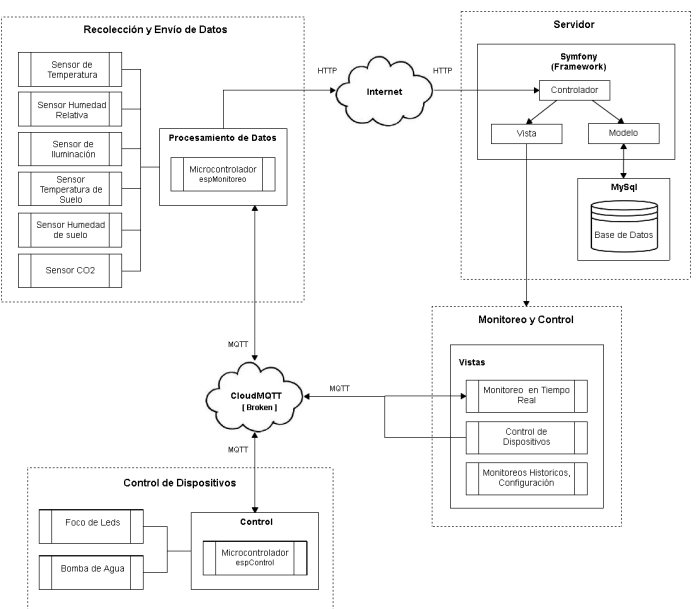

Figura 7. Arquitectura del sistema.

Cada uno de los módulos descritos en la Figura 7 y que forman parte del sistema se describen en la Tabla 8.

\section{Diagrama parcial de caso de uso del sistema}

Un diagrama de caso de uso es una forma de definir las interacciones que se desarrollan entre un sistema y sus actores. Para este caso, los actores involucrados en el sistema se pueden observar en la Figura 8, interactuando con los módulos administrador y usuario. 
Tabla 8. Módulos del sistema propuesto.

\begin{tabular}{|c|c|}
\hline Módulos & Descripción \\
\hline $\begin{array}{l}\text { Recolección } \\
\text { y envío de } \\
\text { datos }\end{array}$ & $\begin{array}{l}\text { Involucra un microcontrolador (espMonitoreo) con acceso a internet y algunos sensores, con } \\
\text { los que obtiene ciertos datos del medio ambiente que lo rodea. Este microcontrolador posee un } \\
\text { proceso ejecutándose, quien cada cierto tiempo permite la extracción y manipulación de los } \\
\text { datos desde los sensores, para luego enviarlos al servidor de la aplicación. Dentro de este mismo } \\
\text { proceso el microcontrolador solicita al servidor los niveles máximos y mínimos registrados } \\
\text { por el usuario, para luego compararlos con los datos obtenidos desde los sensores y si alguno } \\
\text { sobrepasa dichos niveles, este se comunica con otro microcontrolador, el que está a cargo del } \\
\text { control de dispositivos, para que encienda o apague el foco de leds. El microcontrolador también } \\
\text { actúa como servidor cuando un cliente solicita que se le envíen los datos capturados desde los } \\
\text { sensores en tiempo real. }\end{array}$ \\
\hline Servidor & $\begin{array}{l}\text { a aplicación en sí, que está a cargo de escuchar y recibir los datos enviados por el microcontrolador } \\
\text { recolección, e insertarlas en la base de datos MySql. Además es la encargada de enviar la } \\
\text { formación solicitada a la vista del usuario o administrador cuando este la requiera. }\end{array}$ \\
\hline $\begin{array}{l}\text { Control de } \\
\text { dispositivos }\end{array}$ & $\begin{array}{l}\text { Involucra un microcontrolador (espControl) que controla los dispositivos ubicados en el } \\
\text { invernadero como el foco de leds, el que se prende como una señal de alarma cuando el módulo } \\
\text { de recolección y envío de datos detecta que los niveles sensados están sobrepasando los niveles } \\
\text { máximos y mínimos permitidos, y la bomba de agua que es activada por el usuario desde la } \\
\text { web para regar los cultivos dentro del invernadero. }\end{array}$ \\
\hline $\begin{array}{l}\text { Monitoreo y } \\
\text { Control }\end{array}$ & $\begin{array}{l}\text { Son las vistas que muestran el histórico de los datos del medio ambiente recolectados hasta la } \\
\text { fecha y la opción de configurar ciertos parámetros. Por otro lado, muestran los datos obtenidos } \\
\text { por los sensores en tiempo real y permite controlar los dispositivos que están a cargo del } \\
\text { microcontrolador de control. }\end{array}$ \\
\hline CloudMQTT & $\begin{array}{l}\text { Es el servidor de mensajería que actúa como el canal de comunicación entre el usuario y los } \\
\text { microcontroladores a cargo de la recolección de datos y el control de dispositivos. CloudMQTT } \\
\text { actúa como un broker entre estos dispositivos, permitiendo una interacción en tiempo real. }\end{array}$ \\
\hline
\end{tabular}

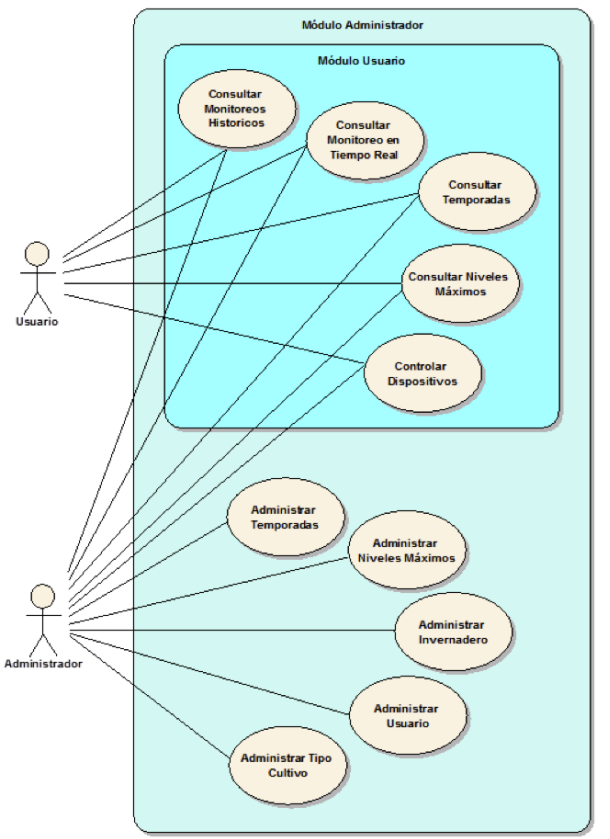

Figura 8. Caso de uso módulos usuario y administrador.
La Tabla 9 describe los casos de uso del módulo administrador, el que a su vez también abarca los del módulo usuario.

\section{Modelo lógico relacional}

Para el desarrollo del sistema se han definido seis entidades para la persistencia de los datos. La interacción de estos, se puede ver en la Figura 9, quienes muestra el diagrama lógico relacional. En la Tabla 10, se describen las distintas entidades.

\section{IMPLEMENTACIÓN}

Para el desarrollo del sistema se utilizó una variedad de tecnologías tanto para la parte del monitoreo y control del sistema, como para las interfaces web. Para el monitoreo y control de dispositivos se utilizaron placas NodeMCU, las que están basadas en el módulo Esp8266 y pueden ser programadas igual que una placa Arduino, mediante el IDE y 
Tabla 9. Descripción de los casos de uso del módulo administrador.

\begin{tabular}{|l|l|}
\hline \multicolumn{1}{|c|}{ Caso de Uso } & \multicolumn{1}{c|}{ Descripción } \\
\hline $\begin{array}{l}\text { Consultar monitoreo } \\
\text { histórico }\end{array}$ & $\begin{array}{l}\text { El usuario o administrador puede consultar el histórico de los monitores de una } \\
\text { determinada variable y observarlas mediante el despliegue de un gráfico. }\end{array}$ \\
\hline $\begin{array}{l}\text { Consultar monitoreo en } \\
\text { tiempo real }\end{array}$ & $\begin{array}{l}\text { El usuario o administrador puede consultar las lecturas que se obtienen desde los } \\
\text { sensores en ese instante, dentro del invernadero seleccionado y recibe unos gráficos } \\
\text { en forma de reloj con las lecturas de los sensores en tiempo real. }\end{array}$ \\
\hline Consultar temporadas & $\begin{array}{l}\text { Permite al usuario o administrador consultar por todas las temporadas registradas } \\
\text { dentro de un invernadero seleccionado. }\end{array}$ \\
\hline $\begin{array}{l}\text { Consultar niveles } \\
\text { máximos }\end{array}$ & $\begin{array}{l}\text { El usuario o administrador puede consultar los niveles máximos y mínimos registrados } \\
\text { hasta la fecha, para las distintas variables climáticas dentro de la temporada seleccionada. }\end{array}$ \\
\hline Controlar dispositivos & $\begin{array}{l}\text { Permite al usuario o administrador controlar dispositivos como alarma y bomba de } \\
\text { agua, ubicados dentro del invernadero seleccionado, encendiéndolos o apagándolos } \\
\text { desde la interfaz web y en tiempo real. }\end{array}$ \\
\hline Administrar temporadas & $\begin{array}{l}\text { Permite al administrador administrar las temporadas de cultivo, como crear una } \\
\text { nueva para un determinado invernadero, editar uno ya existente o eliminarla si es } \\
\text { que esta todavía no contiene lecturas registradas. }\end{array}$ \\
\hline $\begin{array}{l}\text { Administrar niveles } \\
\text { máximos }\end{array}$ & $\begin{array}{l}\text { El administrador puede crear nuevos niveles máximos y mínimos para una determinada } \\
\text { variable climatológica dentro de una temporada seleccionada o editar una ya existente. }\end{array}$ \\
\hline Administrar invernadero & $\begin{array}{l}\text { Permite al administrador crear un nuevo invernadero, modificar uno ya existente o } \\
\text { eliminarlo si no contiene temporadas ni lecturas registradas. }\end{array}$ \\
\hline Administrar usuario & $\begin{array}{l}\text { El administrador puede crear un nuevo usuario, modificar los datos de uno ya } \\
\text { existente o eliminarlo del sistema. }\end{array}$ \\
\hline Administrar tipo cultivo & $\begin{array}{l}\text { Permite al administrador crear un nuevo cultivo, modificar los datos de uno ya } \\
\text { existente o eliminarlo del sistema si no está asociado a ninguna temporada. }\end{array}$ \\
\hline
\end{tabular}

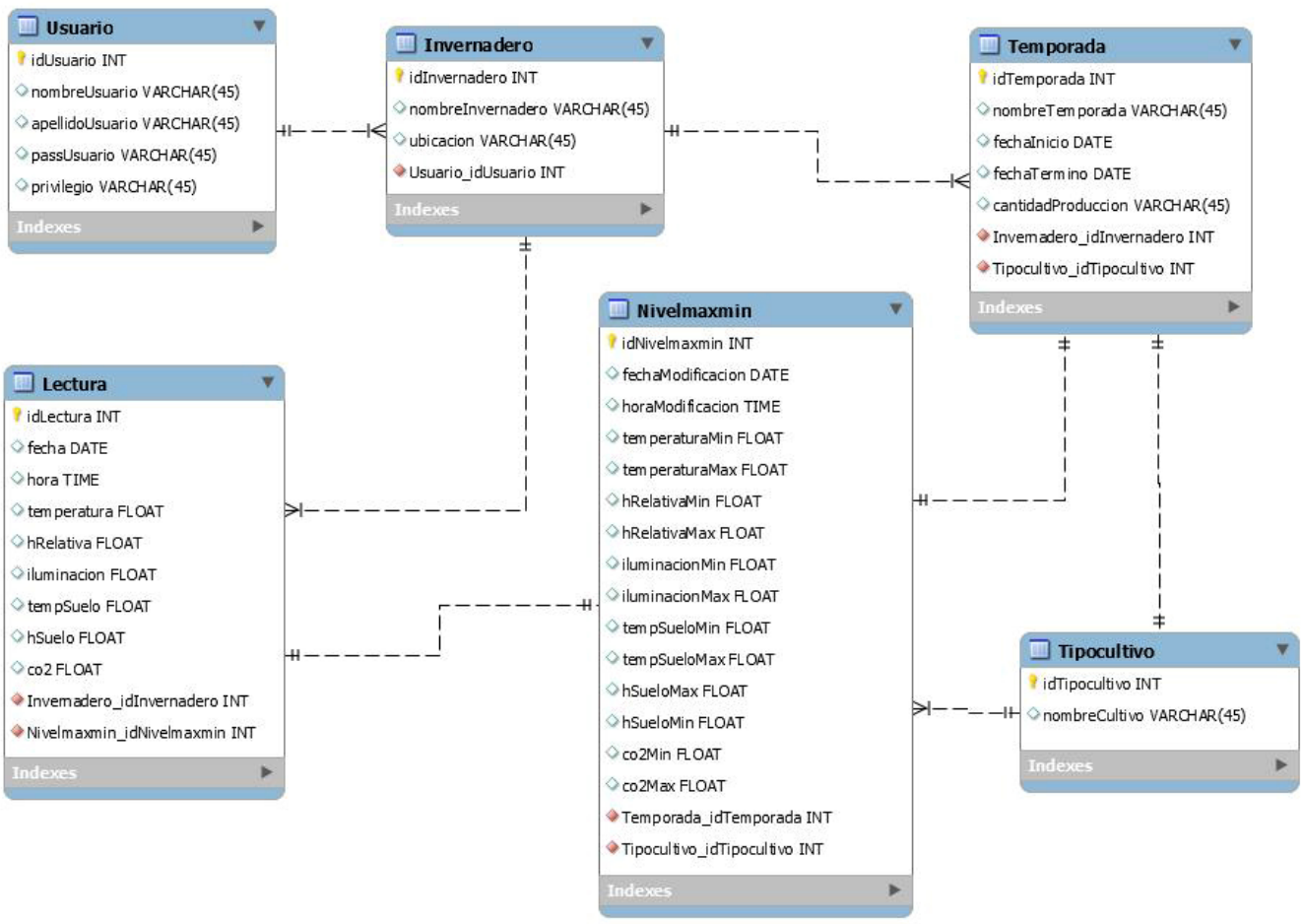

Figura 9. Diagrama lógico relacional. 
Tabla 10. Descripción de tablas de datos operados por el sistema.

\begin{tabular}{|c|c|}
\hline Tabla & Descripción \\
\hline Usuario & $\begin{array}{l}\text { Es la persona a cargo de uno o varios } \\
\text { invernaderos, posee permisos para } \\
\text { ingresar al sistema web de monitoreo, } \\
\text { ya sea como usuario solo para observar } \\
\text { la información que se muestra o como } \\
\text { administrador para realizar algunas } \\
\text { modificaciones en este. }\end{array}$ \\
\hline Invernadero & $\begin{array}{l}\text { Es el recinto cerrado donde se cultiva, } \\
\text { este está bajo el cuidado de un usuario, } \\
\text { posee varias temporadas de cultivo y } \\
\text { muchas lecturas con respecto al clima } \\
\text { dentro de este. }\end{array}$ \\
\hline Temporada & $\begin{array}{l}\text { Es el período de tiempo en el que } \\
\text { se desarrolla un cultivo, este abarca } \\
\text { desde su plantación hasta su cosecha. }\end{array}$ \\
\hline Lectura & $\begin{array}{l}\text { Son los valores de temperatura, } \\
\text { humedad relativa, iluminación, } \\
\text { temperatura de suelo, humedad de } \\
\text { suelo y dióxido de carbono, tomados } \\
\text { desde un invernadero a una cierta hora. }\end{array}$ \\
\hline $\begin{array}{l}\text { Nivel Max. } \\
\text { Min. }\end{array}$ & $\begin{array}{l}\text { Son los valores máximos y mínimos } \\
\text { que pueden tomar la temperatura, } \\
\text { humedad relativa, iluminación, } \\
\text { temperatura de suelo, humedad de } \\
\text { suelo y dióxido de carbono, para el } \\
\text { buen desarrollo de un determinado } \\
\text { cultivo, dentro de una temporada. }\end{array}$ \\
\hline Tipo cultivo & $\begin{array}{l}\text { Es el cultivo que se desarrolla dentro } \\
\text { de una temporada, como el tomate, } \\
\text { morrón, lechuga, etcétera. }\end{array}$ \\
\hline
\end{tabular}

el lenguaje propio de este último que es similar a $\mathrm{C} / \mathrm{C}++$ y es del tipo imperativo. Puede ser usado para desarrollar objetos interactivos, puede percibir el entorno tomando entradas de una vasta gama de artefactos, tal como sensores y puede afectar a su alrededor mediante el control de una variedad de luces, motores y otras salidas físicas. En el caso de monitoreo también se utilizaron sensores de temperatura, humedad relativa, iluminación, temperatura de suelo, humedad de suelo y de dióxido de carbono. Para el control de dispositivos se utilizó un foco de leds y una bomba de agua.

Para el desarrollo de aplicación web que se estará ejecutando en la parte del servidor se utilizó un framework de aplicaciones web PHP y Twig, basado en el patrón Modelo Vista Controlador
(MVC), como también javascript, css y $M y S q l$ para la base de datos. El framework que se utilizó fue Symfony, el que está diseñado para optimizar el desarrollo de las aplicaciones web, facilitando su programación, ya que encapsula operaciones complejas en instrucciones sencillas. Se basa en el patrón MVC, separando la lógica de negocio, la lógica de servidor y la presentación de la aplicación. Este framework está desarrollado completamente con PHP y es compatible con la mayoría de gestores de bases de datos, como MySQL, PostgreSQL, Oracle y $S Q L$ Server de Microsoft. Se puede ejecutar en plataformas Unix, Linux, o Windows.

\section{Dispositivos del sistema}

En la Tabla 11, se describen los principales dispositivos seleccionados para el desarrollo de este proyecto.

\section{ENTORNOS DE DESARROLLO}

\section{IDE Sublime Text}

Sublime Text es un editor de código que está escrito en $\mathrm{C}++$ y Python, permite programar en la mayoría de lenguajes de programación y formatos documentales de texto, utilizados en la actualidad: Java, Python, Perl, HTML, JavaScript, CSS, HTML, XML, PHP, C, $C++$, etc. Su interfaz es limpia e intuitiva y soporta el uso de Snippets, Plugins y sistemas de construcción de código. Este editor se distribuye en forma gratuita, pero no es libre. El IDE permite, mediante los atajos de teclado que posee, acceder de una forma rápida a cualquier complemento del programa. Además dependiendo del lenguaje utilizado, el IDE aplica un esquema de color determinado a cada parte del texto del programa.

\section{IDE Arduino}

Arduino posee su propio IDE y lenguaje de programación y como NodeMCU es compatible con estas, Arduino puede ser programado de la misma forma, solo con instalar la librería necesaria en el IDE.

\section{VISTA MÓDULO ADMINISTRADOR}

A continuación se describen las principales vistas del sistema de monitoreo y control vía web, desarrolladas mediante el framework Symfony. Para ello se seleccionaron las vistas del módulo administrador ya que abarca también las del módulo usuario. 
Tabla 11. Descripción de los principales dispositivos del sistema.

\begin{tabular}{|c|c|}
\hline Dispositivo & Descripción \\
\hline Microcontrolador & $\begin{array}{l}\text { Se utiliza la placa NodeMCU, el que está basado en el módulo Esp } 8266 \text { y contiene una serie } \\
\text { de pines de entrada y salida, con estos se puede acceder a internet en forma inalámbrica y } \\
\text { conectar los sensores necesarios, como en la placa Arduino. Además, como es compatible } \\
\text { con el IDE de este último, se puede programar como si fuese la misma placa Arduino, solo } \\
\text { con instalar una librería. }\end{array}$ \\
\hline Sensor AM2301 & $\begin{array}{l}\text { Para medir la temperatura y humedad relativa del aire Inicialmente se utilizó el sensor } \\
\text { DTH21, el que es un dispositivo compuesto por un sensor capacitivo y un termistor, además } \\
\text { de un circuito integrado básico en el interior que hace la conversión de señal analógica a } \\
\text { digital para enviar el valor de la temperatura en distintas escalas y la humedad relativa del } \\
\text { ambiente en porcentaje. Algunas de sus características son: } \\
\text { - Rango de medición de temperatura: } 0 \text { a } 50^{\circ} \mathrm{C} \\
\text { - Precisión de medición de temperatura: } \pm 2,0^{\circ} \mathrm{C} \text {. } \\
\text { - Rango de medición de humedad: } 20 \% \text { a } 90 \% \mathrm{RH} \text {. } \\
\text { - Precisión de medición de humedad: } 4 \% \mathrm{RH} \text {. } \\
\text { El sensor AM2301 es una versión con cable del DHT21, ya que este último viene dentro } \\
\text { de un cuerpo de plástico grande y funciona a } 3-5 \text { volts. Es por ello que para este proyecto } \\
\text { se decidió utilizar este sensor para medir la temperatura y humedad relativa. }\end{array}$ \\
\hline $\begin{array}{l}\text { Sensor de } \\
\text { Iluminación }\end{array}$ & $\begin{array}{l}\text { El módulo BH1750 es un sensor de iluminación digital que mide el flujo luminoso } \\
\text { (iluminancia), posee un conversor interno de 16-bit, por lo que entrega una salida digital. } \\
\text { Esta es una versión mejorada del típico sensor de luz a base de un Foto-Resistor (LDR), el } \\
\text { que simplemente entrega un valor analógico. El BH1750 entrega la intensidad luminosa } \\
\text { directamente en unidades Lux (Lx). Algunas características técnicas del sensor BH1750 son: } \\
\text { - Voltaje de operación: 3V-5V. } \\
\text { - Respuesta espectral similar a la del ojo humano. } \\
\text { - Amplio rango de medición 1-65535 lux. } \\
\text { - Modo de bajo consumo de energía. } \\
\text { - Rechazo de ruido a 50/60 Hz. }\end{array}$ \\
\hline Sensor Sht 10 & $\begin{array}{l}\text { El Sht10 es un sensor que permite medir la temperatura y humedad de suelo, está basado } \\
\text { en el chip suizo SHT10 de la empresa Sensirion, posee una carcasa a prueba de agua el que } \\
\text { deja pasar el aire para poder medir dichas variables, ya que puede ser enterrado en el suelo, } \\
\text { además tiene un cable de un metro de longitud para llevar los datos al punto de lectura y } \\
\text { funciona de } 3 \text { a } 5 \text { volts. El sensor Sht. } 10 \text { permite obtener información de temperatura y } \\
\text { humedad a partir de los pines de reloj y datos, proporcionando una salida digital de los } \\
\text { datos totalmente calibrados. La interfaz en serie de } 2 \text { hilos y regulación de tensión interna } \\
\text { permite la integración del sistema de una forma fácil y rápida. El tamaño pequeño y de } \\
\text { bajo consumo de energía hace que el este sensor sea la mejor elección para las aplicaciones } \\
\text { más exigentes. Algunas características de este sensor son: } \\
\text { - Rango de humedad: } 0 \text { a } 100 \% \text { RH. } \\
\text { - Rango de temperatura: }-40 \text { a } 123^{\circ} \mathrm{C} \text {. }\end{array}$ \\
\hline Sensor MQ135 & $\begin{array}{l}\text { El Mq-135 es un sensor para la detección de gases, utilizado para el control de calidad del } \\
\text { aire y es adecuado para la detección NH3 (amoníaco), alcohol, benceno, humo, } \mathrm{CO}_{2} \text {, etc. } \\
\text { Este sensor tiene la sensibilidad ajustable para tener una lectura adecuada, es de un tamaño } \\
\text { pequeño y muy práctico, que lo hace sencillo de utilizar. Además tiene una salida digital } \\
\text { por medio de un comparador con umbral ajustable y una salida analógica que puede ser } \\
\text { medido con cualquier microcontrolador o tarjeta de desarrollo con ADC. }\end{array}$ \\
\hline $\begin{array}{l}\text { Fuente de } \\
\text { alimentación }\end{array}$ & $\begin{array}{l}\text { Para que Arduino o el chip ESP8266 funcionen, necesitan ser alimentados eléctricamente. } \\
\text { Para el proyecto se usó un cargador solar para celular que sirve para alimentar el Arduino y } \\
\text { el chip esp } 8266 \text {. Este dispositivo se puede cargar directamente al sol o mediante corriente } \\
\text { eléctrica (AC230V). }\end{array}$ \\
\hline
\end{tabular}




\section{Vista monitoreo en tiempo real}

Esta vista permite al administrador o usuario, una vez seleccionado el invernadero, observar en tiempo real el estado de las lecturas de los sensores, localizados dentro de este. Mediante la llegada de los datos desde el Topic suscrito del servidor CloudMqtt, donde dichos datos son publicados por el microcontrolador del módulo de "Recolección y envío de datos" en forma consecutiva.

Como se observa en la Figura 10, al seleccionar el invernadero deseado, se despliegan una serie de gráficos en forma de reloj que muestran la lectura actual desde cada sensor y el valor máximo (último valor en color amarillo) y mínimo (primer valor en color amarillo) que pueden tomar para no generar la alerta.

\section{Vista monitoreo histórico}

En esta vista, al seleccionar el invernadero, la temporada y el tipo de medida, se despliega un gráfico con el historial de todas las lecturas, para la temporada seleccionada. En la Figura 11 se muestra

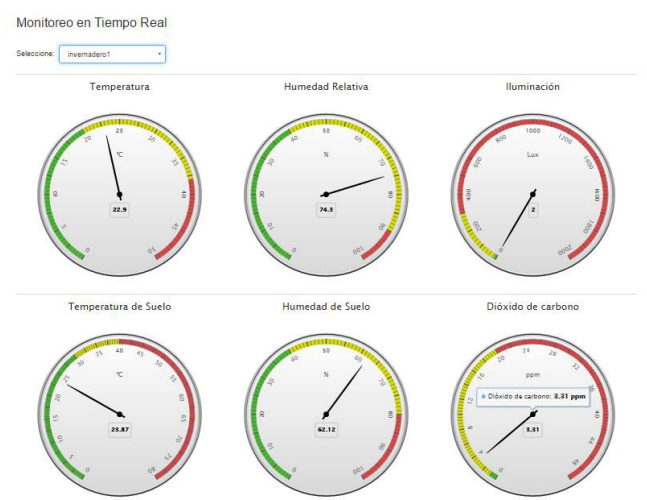

Figura 10. Vista para monitorear en tiempo real.

el gráfico de todas las lecturas de temperatura registradas en la temporada seleccionada, cada una de ellas con la respectiva hora y fecha en que fue tomada, además de los niveles mínimos y máximos permitidos para esa temporada, mediante las línea azul y rojo respectivamente.

También se pueden observar estas lecturas en una forma más detallada, mediante una tabla en una

\section{Monitoreos por temporadas}

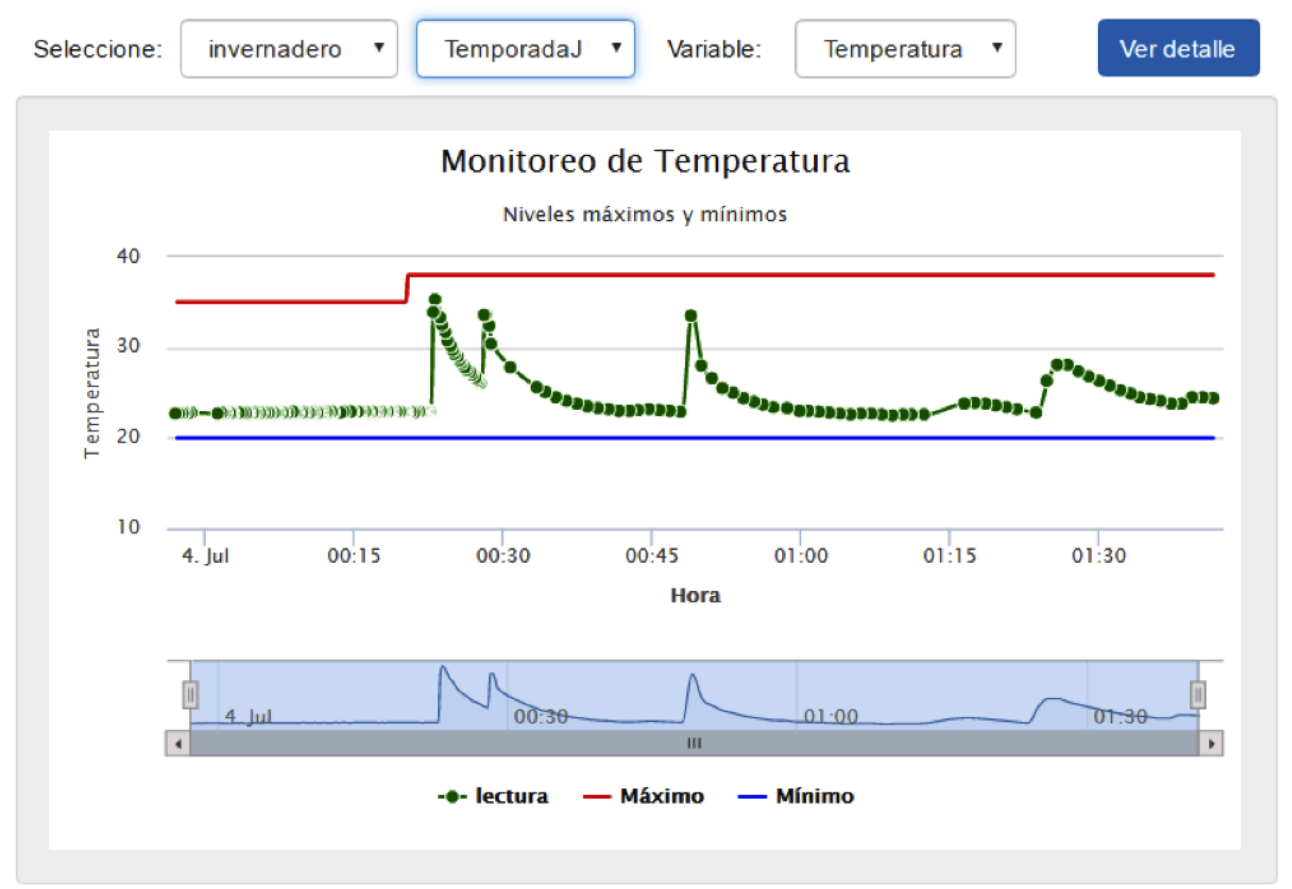

Figura 11. Vista para monitorear el histórico de lecturas. 
ventana desplegable, donde se puede observar la fecha y hora de cada lectura de la variable seleccionada y el valor de esta (Figura 12).

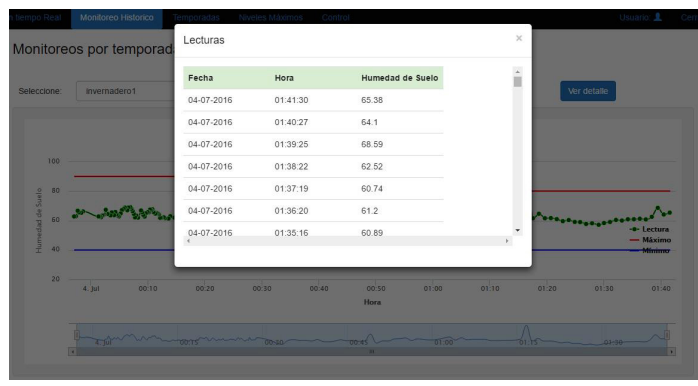

Figura 12. Vista detalle del histórico de lecturas.

\section{Vista temporadas}

Esta vista permite crear una nueva temporada de cultivo para el invernadero seleccionado, así como también observar las ya registradas, mediante una tabla, donde se especifica el nombre de la temporada, su fecha de inicio y término, el cultivo que se plantó y la cantidad de producción que se obtuvo a la hora de la cosecha, los que pueden ser modificados, dado que cada temporada puede ser editada o eliminada. Esta última opción solo si aún no posee lecturas registradas. La Figura 13 muestra un ejemplo de esta vista.

Temporadas por Invernadero

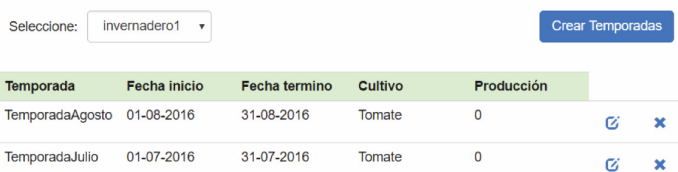

Figura 13. Vista de temporadas creadas por invernadero.

\section{Vista niveles mínimos y máximos}

Cada temporada posee niveles mínimos y máximos para cada variable climatológica, en el que dentro de este rango, el cultivo crece favorablemente. Pero puede darse la necesidad de cambiar alguno de estos valores, debido a que el cultivo en alguna parte de su crecimiento, requiera otro valor máximo o mínimo. Por ello para cada temporada se pueden crear nuevos niveles que son asociados a las lecturas tomadas desde los sensores. Cabe destacar que ningún nivel registrado puede ser eliminado ya que estos, están asociados a lecturas, que al ser analizados, se pueden detectar variaciones que llevarían a tomar determinadas decisiones. En la Figura 14 se muestra el caso de una temporada con más de un registro de niveles máximos y mínimos.

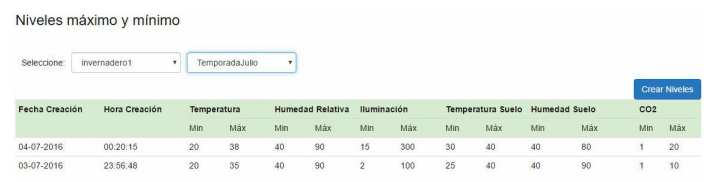

Figura 14. Vista de niveles mínimos y máximos creados por temporada.

\section{Vista control}

En la Figura 15 se muestra la vista de control, que como su nombre lo dice, permite controlar, en este caso una alarma y una bomba. Por un lado, se tiene la alarma que al ser activada produce que se encienda un foco led en el invernadero, anunciando que alguna de las variables climatológicas dentro de él está fuera del rango (nivel mínimo o máximo permitido) para el cultivo que se está desarrollando o se apague la alarma indicando que todo está bien. Por otro lado, se tiene el botón de la bomba que al ser encendido, permite activar la bomba de agua ubicada en el invernadero y se comience a regar los cultivos; y al ser apagada, termine el proceso de regadío.
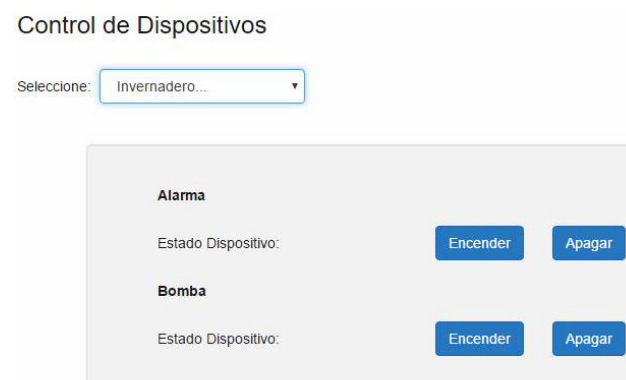

Figura 15. Vista control de dispositivos.

\section{Vista configuración}

Como muestra en la Figura 16, esta vista permite configurar los usuarios con acceso al sistema, invernaderos y tipos de cultivo. 


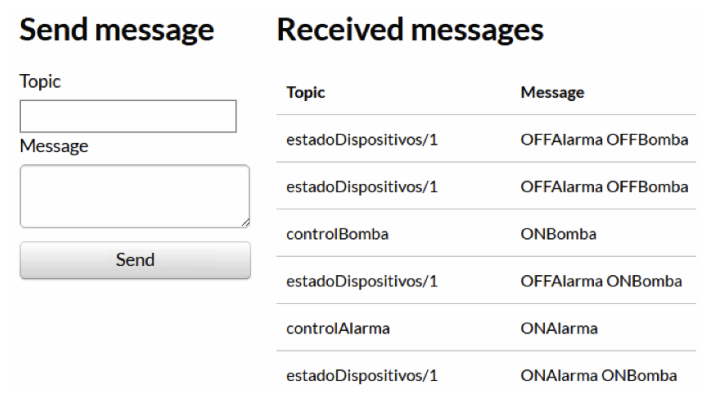

Figura 16. Vista de configuración.

Donde para cada uno de estos casos se pueden observar los ya registrados en el sistema, mediante la lista desplegable, crear uno nuevo, modificar o eliminar.

\section{PRUEBAS DEL SISTEMA}

Un proceso de prueba conlleva la realización de un conjunto de tareas a lo largo del ciclo de vida del sistema. De acuerdo con el estándar IEEE 10122016 [15] el conjunto mínimo de pruebas que se deben realizar son:

- Prueba modular que consiste en la prueba de cada módulo aislado del resto del sistema.

- Prueba de integración que se realiza a medida que los diferentes módulos del sistema se integran en el mismo. Ya se ha realizado la prueba modular, y se supone que todos los módulos son correctos. El objetivo fundamental de esta prueba es comprobar que las interfaces entre los distintos módulos son correctas.

- Prueba del sistema que se realiza cuando se han integrado todos los módulos, y su objetivo es comprobar que el sistema satisface los requisitos del usuario, tanto los funcionales como los no funcionales.

- Prueba de aceptación que se realiza una vez que el sistema se ha implantado en su entorno real de funcionamiento, y su objetivo es demostrar al usuario que el sistema satisface sus necesidades.

- Prueba de regresión que tiene como objetivo comprobar que toda nueva versión de un producto software es de no menos calidad que la versión anterior, es decir, que al introducir cambios no se ha reducido la valoración de ninguna de las características de calidad que tenía el producto. Esta prueba no es aplicable en este caso dado que es una primera versión.
A continuación se describen las pruebas realizadas sobre el sistema desarrollado.

\section{Prueba modular}

Para este tipo de prueba se verificó que los principales módulos del sistema estén funcionando correctamente, enviando y recibiendo datos.

\section{Módulo recolección y envío de datos}

Para observar y comprobar el funcionamiento de este módulo se conectaron los sensores al microcontrolador espMonitoreo y este último a un computador mediante puerto USB. El microcontrolador se pudo conectar a internet en forma inalámbrica, en este caso a la red Wi-Fi llamada Constanza, y luego al servidor CloudMqtt. Por otro lado, también se tiene el envío de los datos capturados desde los sensores al servidor encargado de guardarlos en una base de datos. Esta tarea a cargo de la función espClient() se ejecutó correctamente ya que el microcontrolador se pudo conectar a dicho servidor y enviarle los datos.

\section{Módulo control de dispositivos}

Para comprobar el funcionamiento de este módulo se conectaron el foco led y la bomba de agua a un pequeño relé que es controlado por microcontrolador espControl y este último a un computador mediante puerto USB. En primer lugar el microcontrolador se pudo conectar a internet mediante la red Constanza y luego al servidor CloudMqtt. Una vez conectado a este el microcontrolador comienza a enviar mediante la función estadoPublish() el estado de los dispositivos que controla los que inicialmente están apagados, pero en un momento dado el microcontrolador recibe desde el servidor ClouMqtt la orden de encender la bomba, estas realiza en forma inmediata mediante la función estadoPublish() y continúa enviando el estado de los dispositivos, pero ahora avisando que la bomba está encendida. Luego el microcontrolador recibe otra orden desde el servidor CloudMqtt, en este caso la de encender la alarma (foco led) lo que realiza en forma inmediata y continua enviando el estado de los dispositivos que ahora ambos están encendidos.

\section{Prueba de integración}

En este tipo de prueba se integraron los módulos descritos en la sección anterior con otros del sistema. 
Integración módulo recolección y envío de datos Al integrar el módulo de "recolección y envió de datos" con el servidor CloudMqtt se comprueba que la función sendDatosPublish() logró subir los datos a internet para su lectura en tiempo real. Se pudo observar que los datos enviados por el microcontrolador espMonitoreo son recibidos correctamente por el servidor CloudMqtt en un Topic determinado y a su vez son recibidos en la interfaz de la aplicación web como muestra la Figura 17.

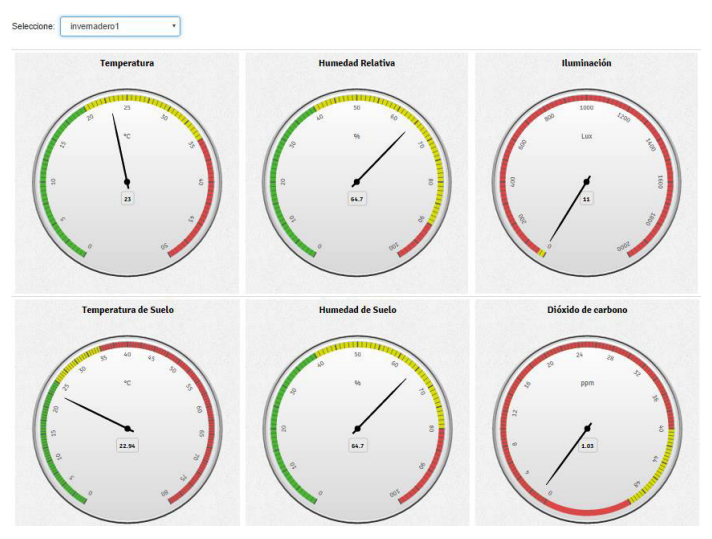

Figura 17. Datos obtenidos desde los sensores en tiempo real.

El módulo de "recolección y envió de datos" también se integró con el módulo servidor que tiene acceso a la base de datos y donde se encuentra la aplicación web del sistema. De esto se logró que los datos enviados por el espMonitoreo fuesen recibidos por el servidor mediante la función cargabdAction() del controlador Lectura y guardados en la base de datos.

\section{Integración módulo control de dispositivos}

$\mathrm{Al}$ integrar este módulo con el servidor CloudMqtt se logró subir el estado de los dispositivos a internet para su revisión en tiempo real. Por otro lado, también se logró enviar desde el servidor de la aplicación web, mediante la interfaz del usuario, la orden para encender o apagar alguno de los dispositivos, como se puede observar en la Figura 18.

Como se puede apreciar en la Figura 19 el estado de los dispositivos aparece en la interfaz del usuario al seleccionar el invernadero relacionado a estos y al presionar sobre alguna de las opciones.

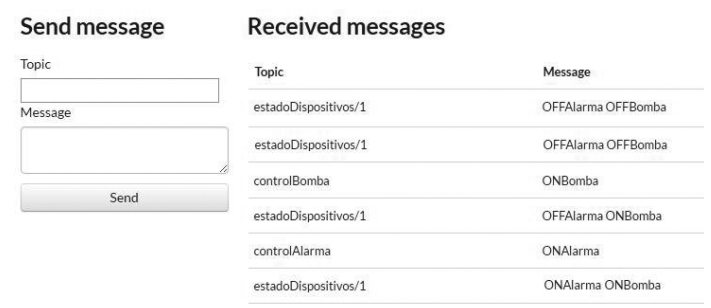

Figura 18. Mensajes enviados desde y hacia el microcontrolador.

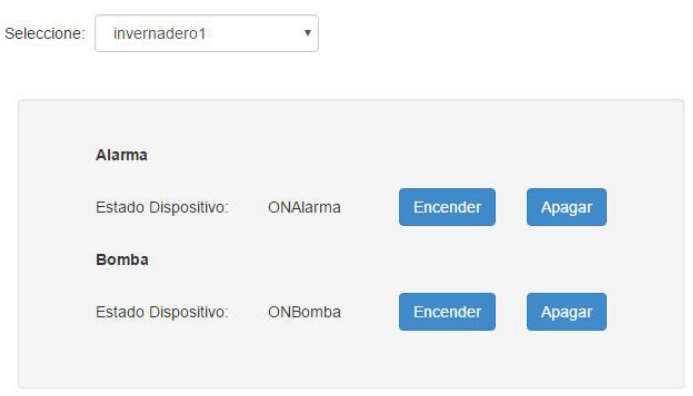

Figura 19. Interfaz de control de dispositivos.

\section{Prueba de sistema}

Para este tipo de prueba se llevó el sistema a uno de los laboratorios de la Facultad de Agronomía de la Universidad de Tarapacá, específicamente al laboratorio de "Cultivos de Tejidos Vegetales", donde se requiere llevar un permanente y estricto control de las variables ambientales para los procesos fisiológicos de los tejidos y células de las plantas que se mantienen en cultivo dentro de tubos de ensayo. Dentro del laboratorio, por un lado, se instaló el circuito eléctrico basado en el microcontrolador espMonitoreo y sensores, como muestra la Figura 20, cuyo funcionamiento fue comprobado desde la interfaz web, donde los datos en tiempo real se recibieron correctamente, ya que, por ejemplo, si se bloqueaba el sensor de luz, este inmediatamente enviaba una lectura de cero lux que significa un ambiente sin iluminación. Además, se pudo comprobar que las lecturas tomadas eran las correctas como es el caso de la temperatura corroborada mediante otro dispositivo, propio del laboratorio, como muestra la Figura 21.

Por otro lado tal como se muestra en la Figura 22 dentro del laboratorio se instaló el circuito eléctrico basado en el microcontrolador espControl que 


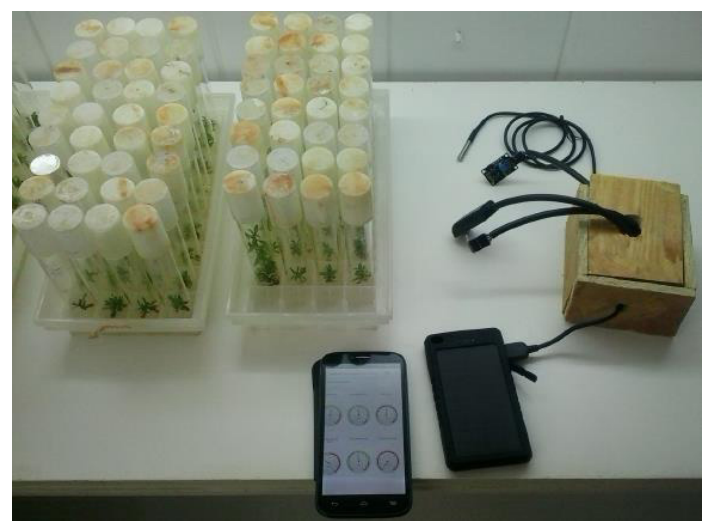

Figura 20. Lecturas en tiempo real.

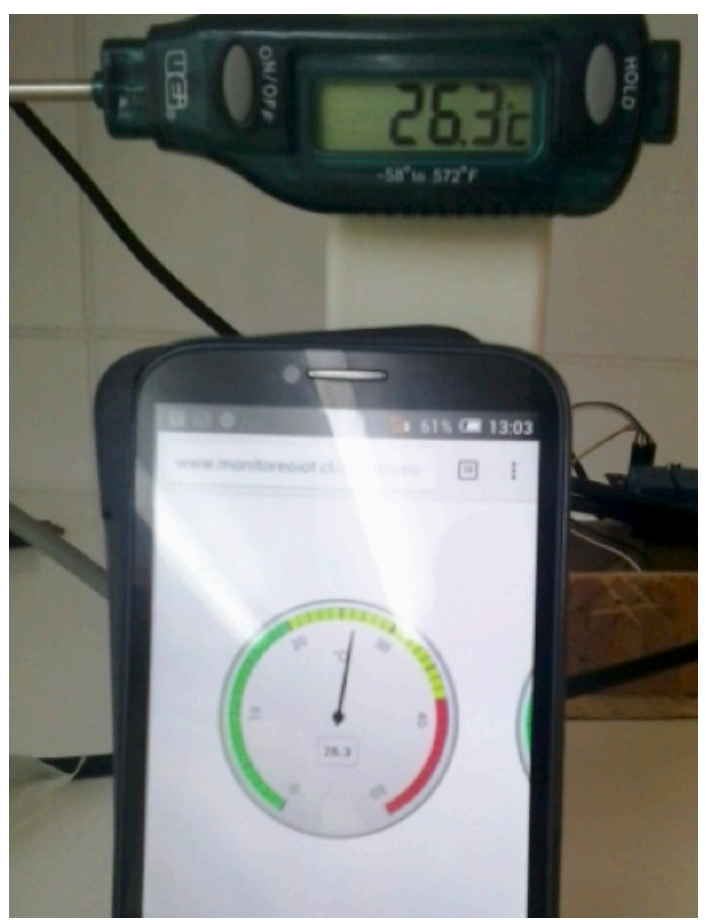

Figura 21. Corroborando temperatura en tiempo real.

puede estar conectado a cualquier dispositivo de 220 volts y controlarlo.

Para este caso se conectó un par de tubos fluorescente del laboratorio que permiten dar iluminación a las plantas. Estos fueron encendidos y apagados desde la interfaz web y su estado era actualizado inmediatamente como muestran las Figuras 23 y 24.

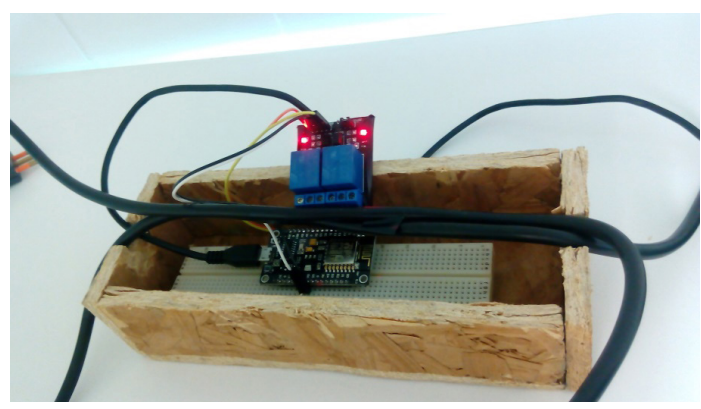

Figura 22. Circuito basado en el microcontrolador espControl.

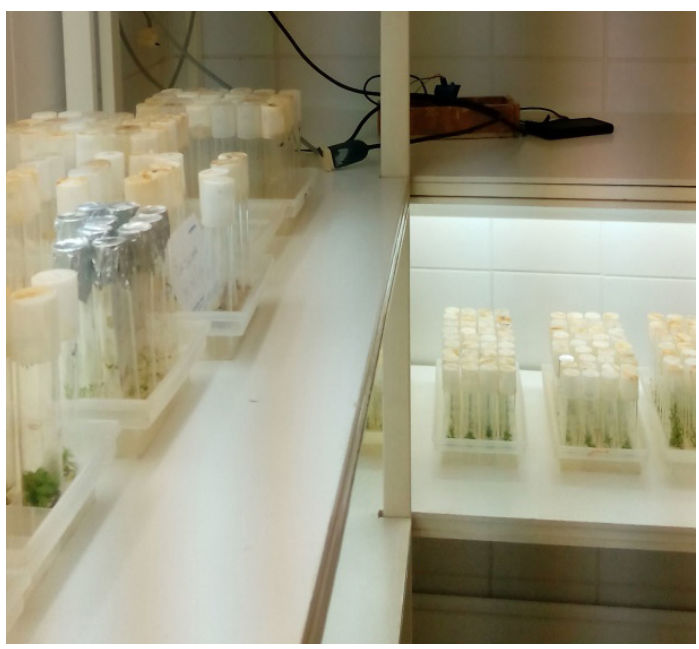

Figura 23. Encendido de tubos fluorescentes.

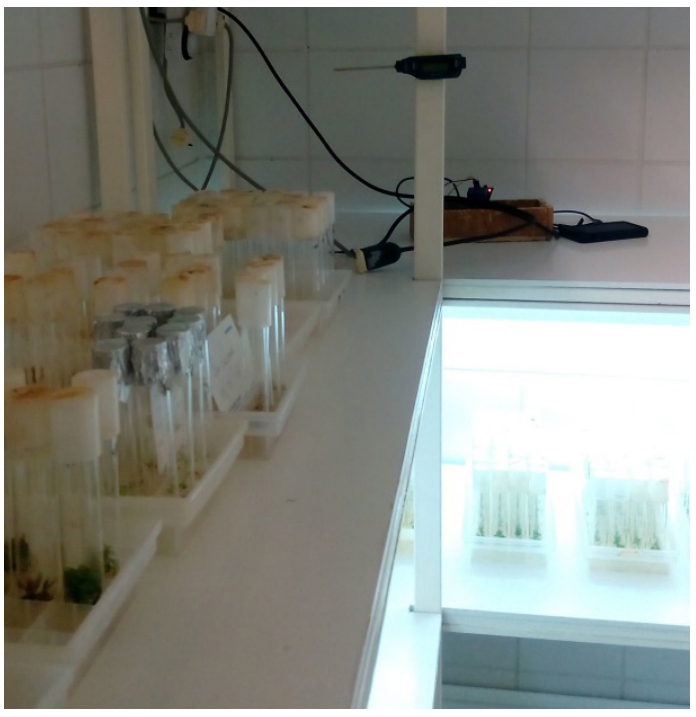

Figura 24. Apagado de tubos fluorescentes. 


\section{Prueba de aceptación}

Para este caso de prueba, el usuario a quien se le presento el sistema fue el jefe de carrera de la Facultad de Agronomía, el quienes lo revisó y estuvo presente en la prueba dentro del laboratorio, quedando satisfecho con los resultados, donde él pudo comprobar en directo el control de la luz y el monitoreo del medio ambiente, quienes se comportaban como se esperaba, comentando que "la aplicación de estas tecnologías resultan de especial relevancia para el trabajo cotidiano en este tipo de laboratorio", esto porque, "este sistema ayudaría mucho a llevar el control medioambiental el cual hasta ahora es realizado en forma manual".

\section{RESULTADOS}

\section{Pruebas}

Como se describió en la sección de pruebas, las tecnologías emergentes que componen el sistema en su conjunto funcionaron eficientemente. Los controladores y sensores seleccionados se ajustaron a las necesidades requeridas, dado que se pudieron conectar a internet y permitieron capturar las variables ambientales, además de ser pequeños y económicos. Por otro lado, la implementación de los circuitos electrónicos donde se integraron estos dispositivos, funcionando como se esperaba y permitiendo llevar a cabo las tareas de "recolección y envío de datos" y "control de dispositivos".

Con respecto a la aplicación web del sistema, esta funcionó correctamente ya que se pudo comunicar con los circuitos electrónicos, permitiendo observar desde internet y en tiempo real los datos obtenidos por los sensores y el control de los dispositivos.

Cuando el sistema se probó en un ambiente real, como se vio en la sección pruebas, este funcionó correctamente, entregando los valores de las variables del medio ambiente del lugar y permitiendo controlar ciertos dispositivos que allí se encontraban.

También se realizó un análisis de los resultados obtenidos, donde se pudo comprobar que se cumplió el objetivo planteado para este trabajo.

\section{Comparativas}

Con la finalidad de comparar los sistemas vistos en la sección de los trabajos relacionados con el sistema descrito en este artículo, se establecieron dos enfoques. El primero consiste en analizar las tecnologías utilizadas por los distintos trabajos relacionados estudiados. El segundo dice relación con los costos asociados a los dispositivos utilizados en su implementación.

\section{Tecnologías}

Respecto al factor comparativo tecnológico usado: sensores, microcontroladores, tipo de conexión a internet, mecanismos de comunicación y el alcance de la red; en la Tabla 12 se muestran los resultados de su respectiva comparación.

Como se puede observar desde dicha tabla, las tecnologías seleccionadas para este sistema poseen ciertas ventajas frente a los dispositivos utilizados en los proyectos relacionados, ya que en el caso del

Tabla 12. Sistemas trabajos relacionados vs Sistemas propuesto.

\begin{tabular}{|l|l|l|}
\hline Factor comparativo & \multicolumn{1}{|c|}{ Trabajos relacionados } & \multicolumn{1}{c|}{ Sistemas propuestos } \\
\hline Sensor & $\begin{array}{l}\text { En general, son tratados como nodos } \\
\text { basados en un módulo ZigBee que es de } \\
\text { un costo considerable. }\end{array}$ & $\begin{array}{l}\text { Posee 4 sensores que son pequeños y } \\
\text { económicos. }\end{array}$ \\
\hline Micro controlador & $\begin{array}{l}\text { En general son de variada procedencia, } \\
\text { tamaño y rendimiento, pero de alto costo }\end{array}$ & Placa NodeMcu, pequeña y de un bajo costo. \\
\hline Conexión a internet & $\begin{array}{l}\text { En general utilizan módulos de baja } \\
\text { velocidad, alto costo e inalámbricamente. }\end{array}$ & Se conecta inalámbricamente. \\
\hline Comunicación & $\begin{array}{l}\text { En general utilizan módulos adicionales } \\
\text { (GPRS, NRF2401L, GSM) para conexión } \\
\text { inhalámbrica. }\end{array}$ & $\begin{array}{l}\text { La placa NodeMcu se comunica en forma } \\
\text { inalámbrica y directa con otras placas y } \\
\text { con el servidor en internet. }\end{array}$ \\
\hline Alcance de la red & $\begin{array}{l}\text { En general el alcance es amplio, pero el } \\
\text { servidor dentro de la red local. }\end{array}$ & $\begin{array}{l}\text { El alcance es amplio y el servidor está } \\
\text { en internet. }\end{array}$ \\
\hline
\end{tabular}


microcontrolador de la placa NodeMcu, no necesita estar conectado a ningún dispositivo o módulo extra para conectarse a internet y comunicarse en forma inalámbrica, con el que se pueden desarrollar sistemas de automatización ya que los sensores utilizados son pequeños y económicos.

\section{Costos}

En la Tabla 13 se muestra los costos aproximados de los dispositivos utilizados en cada uno de los trabajos relacionados y del trabajo propuesto, este último al final de la tabla.

Como se puede observar en la tabla, el trabajo propuesto frente a los demás, presenta un menor costo total en los dispositivos utilizados (aunque ofrece un mayor número de sensores con la posibilidad de medir hasta seis tipos de variables climatológicas). Más específicamente, en comparación con el trabajo de Guofang et al. [12] que es la más cercana, es un 42,46\% más económica. Respecto de la más lejana [13], es un 90,42\% más económica.

\section{CONCLUSIONES}

Con la idea de desarrollar un sistema que permitiera llevar a cabo un monitoreo ambiental y control dentro de invernaderos vía web, es que se decidió crear este proyecto, el que fue desarrollado con distintas tecnologías, tanto del área de la electrónica como de la informática.

Para este sistema se seleccionaron dispositivos electrónicos que la actualidad son emergentes, poco explotados y con estos se pueden desarrollar una variedad de proyectos. Esto, debido a las características que poseen, como es el caso de los microcontroladores seleccionados, quienes en comparación a otros no requieren módulos extras, por ejemplo, para conectarse a internet ya que integran todo en una sola placa y cada vez son más pequeños y adaptables para desarrollar cualquier proyecto.

También se seleccionaron tecnologías del área de la informática que están surgiendo de la mano de las tecnologías electrónicas que buscan facilitar las tareas de automatización, como es el caso del protocolo Mqtt.

Al integrar las tecnologías descritas anteriormente, se generó un sistema que demostró ser eficaz y necesario su uso en lugares donde se requiere llevar un control preciso de las condiciones ambientales.

Mediante el desarrollo de este proyecto, se interiorizó en áreas como la agricultura y electrónica donde, en la primera, se aprendió sobre las necesidades, medioambientales para el buen desarrollo de un cultivo y, en la segunda, se aprendió a trabajar con tecnologías propias del área de la electrónica. Al ser "combinadas" con la informática, permiten automatizar distintas áreas mediante el desarrollo de variados sistemas.

La implementación de este sistema fue desarrollada para que el usuario pueda controlar los dispositivos solo al accionar los botones de encender o apagar desde la interfaz web. Por ello, un trabajo a futuro podría ser desarrollar un sistema de control de horarios para que el usuario pueda programar desde internet el accionamiento de los dispositivos de control, para que estos se enciendan o apaguen

Tabla 13. Dispositivos utilizados vs Costos (US\$).

\begin{tabular}{|l|c|c|c|c|}
\hline \multirow{2}{*}{ Trabajo } & \multicolumn{3}{|c|}{ Tecnologías } & \multirow{2}{*}{ Costo total } \\
\cline { 2 - 4 } & Micro controlador & Sensor & Comunicación & \\
\hline Jianfa et al. [9] & 45,64 & 25,86 & 136,98 & 208,48 \\
\hline Tongtong et al. [10] & 82,19 & 9,13 & 138,50 & 229,82 \\
\hline Balaji et al. [11] & 63,92 & $136,93^{*}$ & 38,05 & 238,90 \\
\hline Guofang et al. [12] & 9,13 & 82,95 & 44,90 & 136,98 \\
\hline Collins et al. [13] & 4,56 & 592,84 & 225,26 & 822,66 \\
\hline Mendoza [14] & 38,05 & 45,66 & 114,15 & 197,86 \\
\hline Propuesto & 13,69 & 65,14 & $0^{* *}$ & 78,83 \\
\hline
\end{tabular}

* Utiliza dos pero no están especificados (se usa el promedio).

** Incluido en el microcontrolador. 
a una hora determinada. También este sistema podría ser desarrollado para dispositivos móviles mediante Android.

Finalmente, la propuesta resultó más económica hasta un $90,42 \%$ con respecto a la solución más cara propuesta por otros autores.

\section{AGRADECIMIENTOS}

Este trabajo cuenta con el financiamiento para la Investigación de la Universidad de Tarapacá, Arica, Chile, 2016, en el contexto de proyecto "Monitoreo ambiental de invernadero vía web" (código 872516). Asimismo, al Dr. Hugo Escobar, jefe del laboratorio de Cultivo de Tejidos Vegetales y jefe de Carrera de Agronomía, Facultad de Ciencias Agrarias de la misma Universidad, por su valiosa colaboración, interés y disposición en las pruebas in situ del sistema.

\section{REFERENCIAS}

[1] P. Lombardi. Salto tecnológico en Azapa y Lluta: Mallas antiáfidos, portainjertos de hortalizas y el boom de las semilleras en Arica, 04 Marzo 2016, URL: http:// www.redagricola.com/reportajes/hortalizas/ mallas-antiafidos-portainjertos-de-hortalizasy-el-boom-de-las-semilleras-en-a

[2] Instituto Nacional de Estadísticas, 4 marzo 2016, URL: http://www.ine.cl/canales/menu/ publicaciones/calendario_de_publicaciones/ xls/03_Tabulados_Hortalizas_2014_ publicado(20150421)Preliminar.xlsx.

[3] F. Fabres. Agricultura protegida de Arica un modelo propio en construcción acelerada, 29 octubre 2015, URL: http://www. redagricola.com/reportajes/hortalizas/ agricultura-protegida-de-arica-un-modelopropio-en-construccion-acelerada.

[4] Instituto de Investigaciones Agropecuarias, 4 marzo 2016, URL: http://www.inia.cl/ wp-content/uploads/2015/07/FICHA-04URURI-Demanda-hidrica.pdf.

[5] P. Mazuela, L. Acuña, M. Álvarez \& A. Fuentes. Producción y calidad de un tomate Cherry en dos tipos de invernadero en cultivo sin suelo. Idesia (Arica). Vol. 28 $\mathrm{N}^{\circ}$ 2, pp. 97-100. 2010. URL: https://dx.doi. org/10.4067/S0718-34292010000200012.
[6] Infoagro, "Control climático en invernaderos ( $1^{a}$ parte)", 18 junio 2015, http://www. infoagro.com/industria_auxiliar/control_ climatico.htm.

[7] J. Sánchez, J. Guzmán, F. Rodríguez. Modelado de la transpiración de un cultivo de tomate bajo invernadero para el diseño de sistemas de control de riego, 17 junio 2015, URL: http://www. researchgate.net/profile/M_Berenguel/ publication/228542062.

[8] A. Alvarado. Monitoreo ("Scouting") y la Agricultura Orgánica, 19 junio 2015, URL: http://academic.uprm.edu/ aalvarado/HTMLobj-181/Scounting_y_ AgricOrganica.pdf.

[9] Jianfa Xia, Zhenzhou Tang, Xiaoqiu Shi, Lei Fan, Huaizhong Li. An environment monitoring system for precise agriculture based on wireless sensor networks, Seventh International Conference on Mobile Ad-hoc and Sensor Networks, Beijing, China, December, 2011.

[10] Tongtong Yin,Wenjie Feng, Zheying Li. Temperature and Humidity Wireless Sensing and Monitoring Systems Applied in Greenhouse, International Conference on Computer Science and Network Technology, Harbin, China, December, 2011.

[11] B. Balaji Bhanu, 2K. Raghava Rao, 3J.V.N. Ramesh, Mohammed Ali Hussain. Agriculture Field Monitoring and Analysis using Wireless Sensor Networks for improving Crop Production, Eleventh International Conference on Wireless and Optical Communications Networks (WOCN), Andhra Pradesh, India, September, 2014.

[12] Li Guofang, Chen Lidong, Qi Yubin, Liu Shengtao, Xue Junyu. Remote Monitoring System of Greenhouse Environment Based on Lab VIEW, International Conference On Computer Design And Appliations (ICCDA 2010), vol. 2, Qinhuangdao, Hebei, China, June. 2010.

[13] F. Collins, D. Orpen, C. Fay, C. Foley, A. Smeaton, D. Diamond. Web-based Monitoring of Year-Length Deployments of Autonomous Gas Sensing Platforms on Landfill Sites, Sensors 2011 IEEE, Limerick, Ireland, October, 2011. 
[14] F. Mendoza. Sistema de monitoreo y control de invernaderos a través de una red inalámbrica mediante un servidor web embebido en microcontroladores de alto rendimiento, 22 junio 2015, URL: http:// ri.uaq.mx/bitstream/123456789/2644/1/ RI002386.pdf.

[15] Estándar IEEE 1012-2016, URL: https://standards.ieee.org/findstds/ standard/1012-2016.html 